\title{
Use of clopidogrel in the reduction of myocardial damage during percutaneous coronary intervention
}

\author{
Arijit Dasgupta \\ Debabrata Mukherjee \\ Gill Heart Institute, University \\ of Kentucky, Lexington, KY, USA
}

Correspondence: Debabrata Mukherjee Gill Heart Institute, Division of Cardiovascular Medicine, University of Kentucky, 900 S. Limestone Street, 326 Wethington Building, Lexington, KY 40536-0200, USA

$\mathrm{Tel}+\mathrm{I} 8593235630$

Fax +I 8593236475

Email mukherjee@uky.edu

\begin{abstract}
It is estimated that approximately a quarter of patients undergoing coronary intervention may have significant post-procedural creatinine (CK)/creatinine kinase myocardial band (CK-MB) elevations and approximately half may have post-procedural troponin elevations. Current data suggest that periprocedural infarction is associated with short-, intermediate-, and long-term adverse outcomes, most notably mortality. This review examines the role of clopidogrel in decreasing periprocedural myonecrosis following percutaneous coronary intervention (PCI). Clopidogrel is an important pharmacologic agent used to reduce myocardial infarction post-coronary intervention as assessed directly by the evaluation of cardiac biomarkers and indirectly by the evaluation of short-term ischemic events. The optimal dose of clopidogrel is considered to be at least $300 \mathrm{mg}$ given 6 to 15 hours prior to PCI but there is considerable evidence to suggest that a loading dose of $600 \mathrm{mg}$ given 2 to 6 hours prior to PCI may be more efficacious in limiting post-coronary intervention events. The benefit obtained from clopidogrel appears independent of and incremental to that of other antiplatelet and antithrombotic agents used during and after coronary intervention. Keywords: percutaneous coronary intervention, myonecrosis, clopidogrel, antiplatelet agents, myocardial infarction
\end{abstract}

\section{Introduction}

Percutaneous coronary intervention (PCI) has become the most common form of coronary revascularization in the United States with greater than 900,000 procedures performed annually. ${ }^{1}$ Although overall a safe procedure, PCI does have multiple associated risks including bleeding, coronary dissection, and abrupt vessel closure. These complications are obviously undesirable and intuitively associated with negative longterm outcomes but an even more frequent and important contributor to the mortality and morbidity associated with PCI is periprocedural myonecrosis and infarction. ${ }^{2}$ The definition of periprocedural myocardial infarction was standardized in 2007 with a post-procedure elevation of cardiac biomarkers more than 3 times the 99th percentile upper reference limit (URL) defined as PCI-related myocardial infarction (MI). ${ }^{3}$ It is estimated that approximately $25 \%$ of patients undergoing PCI have significant postprocedural creatinine $(\mathrm{CK})$ /creatinine kinase myocardial band (CK-MB) elevations and approximately $50 \%$ of patients have significant post-procedural troponin elevations. ${ }^{2}$ Initially, it was felt these elevations were simple enzyme leaks with no long-term implications. Multiple data sets have now definitively demonstrated that periprocedural infarction is associated with short-, intermediate-, and long-term adverse outcomes, most notably mortality. ${ }^{4-7}$ This review examines the role of clopidogrel in decreasing periprocedural myonecrosis following PCI.

\section{Pathophysiology of periprocedural myonecrosis and the role of clopidogrel therapy}

PCI invariably results in mechanical plaque disruption with some degree of associated endothelial injury. Platelet attachment then occurs at the site of endothelial 
injury through platelet surface interactions with the exposed extracellular matrix (ECM) and exposed von Willebrand Factor (vWF). ${ }^{8}$ Following platelet attachment, a series of platelet activating steps occur including the secretion of various secondary messengers including ADP, thromboxane A2, and serotonin. ${ }^{9}$ These messengers result in further autoactivation of platelets and subsequent platelet morphological changes resulting in firm platelet adhesion, activation of thrombin, and eventual full platelet aggregation via the glycoprotein IIb/IIIa integrin receptor. ${ }^{10}$ Local vasoconstriction and inflammation, combined with the accumulation and embolization of platelet aggregates, results in thrombosis, ischemia, and infarction in the microvascular circulation. ${ }^{11}$ Endogenous release of vasodilators such as adenosine is able to compensate to some degree but when sufficient platelets aggregate, they are able to exceed all compensatory mechanisms and the result is periprocedural myonecrosis and/or infarction. This highlights the importance of platelet-dependent processes in the pathophysiology of ischemic complications following PCI.

This importance of platelet activity in post-PCI ischemic complications was clinically demonstrated by the Intracoronary Stenting and Antithrombotic Regimen (ISAR) trial, Full Anticoagulation Versus Ticlopidine plus Aspirin after Stent Implantation (FANTASTIC) trial, and Stent Anticoagulation Regimen Study (STARS) trials. ${ }^{12-14}$ Each trial demonstrated the superiority of an anti-platelet approach (aspirin and/or ticlopidine) over anticoagulation in the reduction of the composite endpoint of cardiovascular (CV) death, MI, or need for recurrent revascularization following stent placement and specific post-procedural outcomes such as early stent thrombosis. These trials helped established the role of anti-platelet therapy in reducing both periprocedural and post discharge ischemic complications following PCI.

Three key classes of anti-platelet agents are used currently in clinical practice: aspirin, intravenous glycoprotein IIb/IIIa inhibitors, and thienopyridines. The thienopyridine class of antiplatelet agents is composed of ticlopidine, clopidogrel, and prasugrel which is under FDA review. These drugs act through non-competitive, irreversible antagonism of the ADP binding site on the P2Y12 receptor on platelets. ${ }^{15}$ Ticlopidine's use was limited by its twice-daily formulation and by the main side effects of occasional neutropenia, and even more rarely thrombotic thrombocytopenia purpura (TTP). ${ }^{15}$ Clopidogrel is a second generation thienopyridine agent with an oral formulation requiring gastrointestinal absorption mediated by the P-glycoprotein efflux pump followed by a 2 step cytochrome $\mathrm{p}-450$ dependent activation resulting in the active metabolite. ${ }^{16}$ The use of clopidogrel in place of ticlopidine began in 2000, after the Clopidogrel Aspirin Stent International Cooperative Study (CLASSICS) trial demonstrated better tolerability profile with no change in the rates of recurrent ischemic events. ${ }^{17}$

\section{Clopidogrel and present data}

In the current era, clopidogrel's important pharmacotherapeutic role has been expanded to include patients undergoing PCI for any indication including non-ST elevation acute coronary syndrome (NSTE ACS), routine elective PCI, and ST elevation myocardial infarction (STEMI). The evidence for this comes from three main trials: Effects of Pretreatment with Clopidogrel and Aspirin Followed by Long-Term Therapy in Patients Undergoing Percutaneous Coronary Intervention: the PCI-CURE Study (PCI-CURE), Early and Sustained Dual Oral Antiplatelet Therapy Following Percutaneous Coronary Intervention: A Randomized Control Trial (CREDO), and Effect of Clopidogrel Pretreatment Before Percutaneous Coronary Intervention in Patients wit ST-Elevation Myocardial Infarction Treated with Fibrinolytics: the PCI-CLARITY Study (PCI-CLARITY). ${ }^{18-20}$ These trials demonstrated the clear and consistent benefit of clopidogrel, specifically clopidogrel pretreatment, in the prevention of ischemic complications post-PCI. These trials also helped clarify the optimal timing of clopidogrel pretreatment in limiting thrombotic and ischemic complications post PCI.

PCI-CURE aimed to investigate whether pretreatment followed by long term administration of clopidogrel with PCI was superior to no clopidogrel pretreatment and short-term clopidogrel therapy in terms of reducing post-PCI ischemic events. The study examined outcomes in 2658 patients with NSTE-ACS enrolled in the original Clopidogrel in Unstable Angina to prevent Recurrent Events (CURE) trial ${ }^{21}$ who underwent angiography and PCI at the discretion of the treating physician. These patients had been randomized at presentation to clopidogrel $(\mathrm{N}=1313)$ or placebo $(\mathrm{N}=1345)$ with a $300 \mathrm{mg}$ loading dose followed by $75 \mathrm{mg}$ daily per CURE trial protocol. Patients received pretreatment with clopidogrel for a mean of 6 days prior to angiography. Following PCI, $>80 \%$ of patients in both groups received open-label thienopyridine for 4 weeks, after which the study drug (placebo versus clopidogrel) was restarted for a mean of 8 months.

The primary endpoint was a composite of CV death, MI, or urgent target-vessel revascularization (TVR) within 30 days of PCI. Although the study did not directly assess for 
periprocedural MI rates through routine screening for post-procedure increases in cardiac enzymes, the results did suggest a marked decrease in this outcome. The study found a significant decrease in the primary composite endpoint in the clopidogrel group versus the placebo group $(4.5 \%$ versus $6.4 \% ; p=0.03)$. This difference was driven mostly by a decrease in the number of MIs $(2.1 \%$ versus $3.8 \%)$ and specifically q-wave MIs $(0.8 \%$ versus $2.4 \%)$ post PCI. CV death and urgent TVR rates were statistically similar between the two groups. This lower rate of the primary endpoint was seen as early as 2 days after PCI implicating a reduction in periprocedural MI and the benefit continued until 30 days (Figure 1). As both groups of patients received clopidogrel post PCI for at least 30 days at equally high rates, the benefit of the marked reduction in periprocedural MI was likely due to the effects of clopidogrel pre-treatment with a $300 \mathrm{mg}$ load followed by a $75 \mathrm{mg}$ daily dose given a mean of 6 days prior to PCI. Thus although this study first highlighted the importance of clopidogrel loading prior to PCI to help decrease PCI-related myonecrosis, the optimal timing of this loading dose was not yet clear.

The CREDO study ${ }^{19}$ randomized 1815 patients undergoing planned PCI to $300 \mathrm{mg}$ clopidogrel loading at presentation $(\mathrm{N}=900)$ versus placebo loading at presentation $(\mathrm{N}=915)$ with subsequent clopidogrel daily in both groups through day 28 . Following this, the clopidogrel loading group continued on clopidogrel from day 29 through 12 months while the placebo group was restarted on placebo. Both groups had routine evaluation for periprocedural MI defined as an elevation in the CK or CK-MB levels to at least 3 times the upper limit of normal (ULN) in 2 samples collected at different sampling times. At 28 days, the composite endpoint of death, MI, and urgent TVR was assessed in both groups. The mean duration between clopidogrel loading and PCI was 9.8 hours but with $51 \%$ of patients receiving their load less than 6 hours before PCI.

Clopidogrel loading was associated with a non-significant decrease in the composite endpoint at 28 days $(6.8 \%$ pretreatment versus $8.3 \%$ no pretreatment, $\mathrm{p}=0.23$, Figure 2 ). For each individual component of the combined end point, there were fewer events in patients receiving clopidogrel pretreatment. When the groups were re-analyzed based on the prespecified loading time intervals of 3 to 6 hours, 6 to 12 hours, and 12 to 24 hours prior to PCI, an important interaction was noted between the timing of pretreatment and reduction in the composite endpoint. Patients receiving clopidogrel between 3 and 6 hours prior to PCI had no benefit compared to placebo whereas patients treated 6 to 12 and 12 to 24 hours before PCI had progressively increased protection from adverse cardiac events at 28 days. Patients who received a $300 \mathrm{mg}$ clopidogrel loading at least 6 hours before PCI experienced a $38.6 \%$ relative reduction in the composite endpoint at 8 days that was of borderline statistical

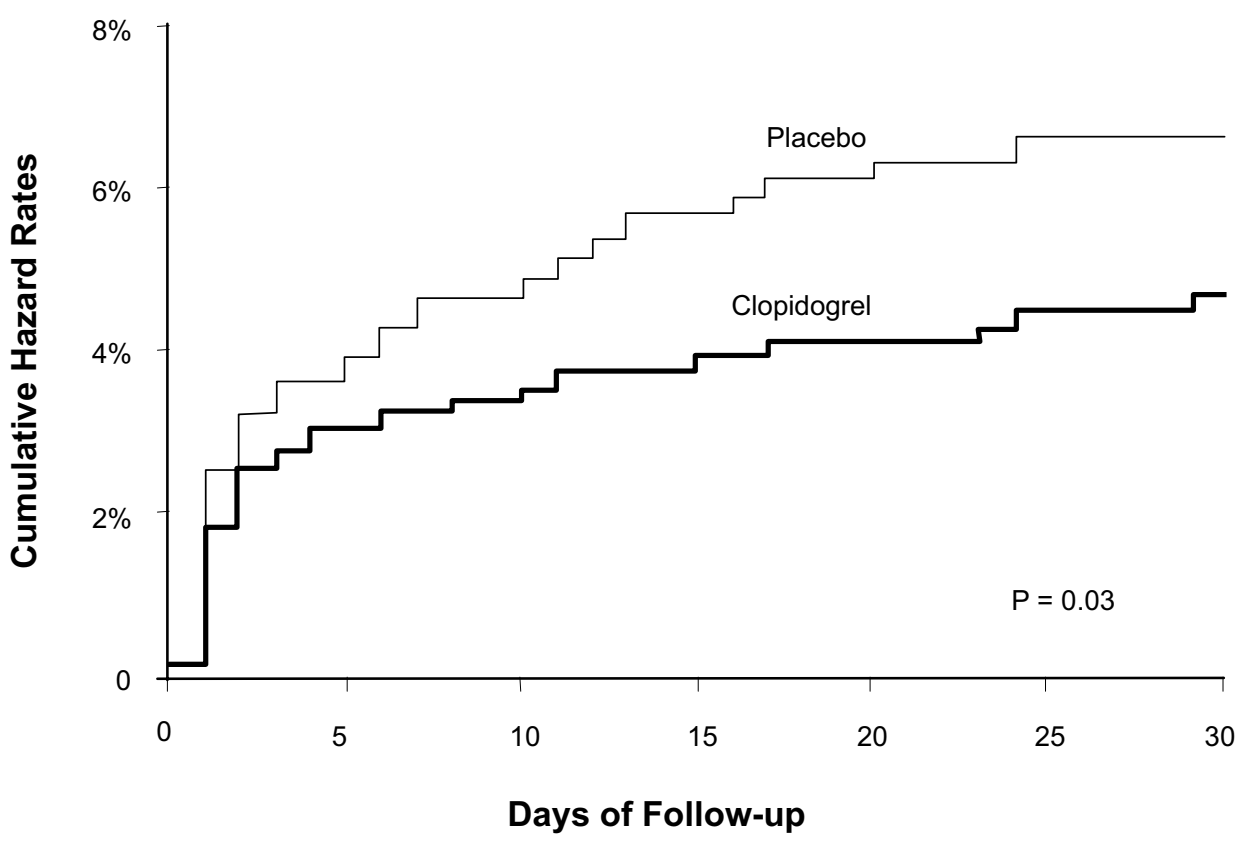

Figure I Kaplan Meier cumulative event rates for primary endpoint at 30 days after PCI in the PCI CURE study. Reprinted from The Lancet, 358, Mehta SR, Yusuf S, Peters RJ, et al. Effects of pretreatment with clopidogrel and aspirin followed by long-term therapy in patients undergoing percutaneous coronary intervention: the PCl-CURE study, $527-533,{ }^{18}$ Copyright (C) 200I, with permission from Elsevier. 


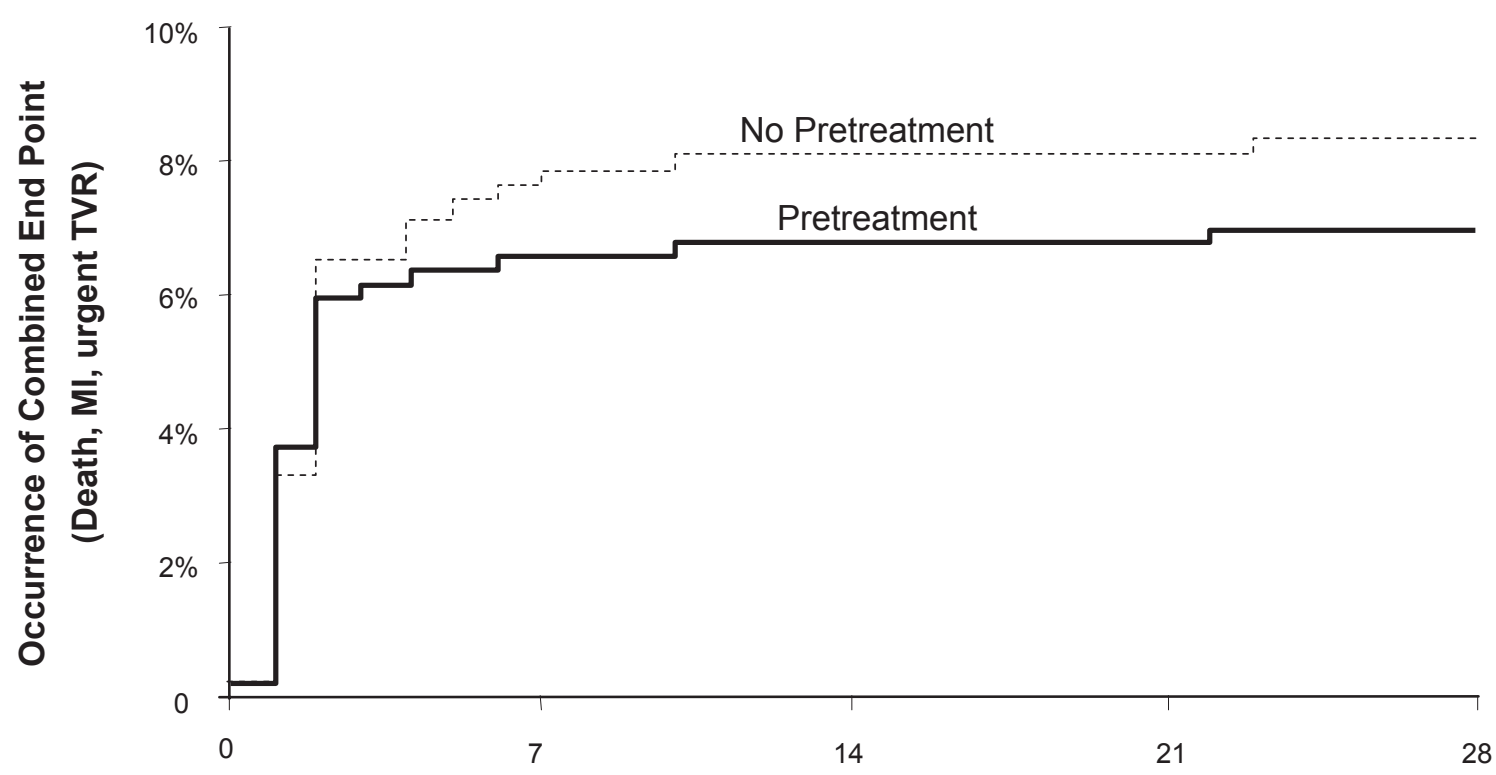

Days from randomization

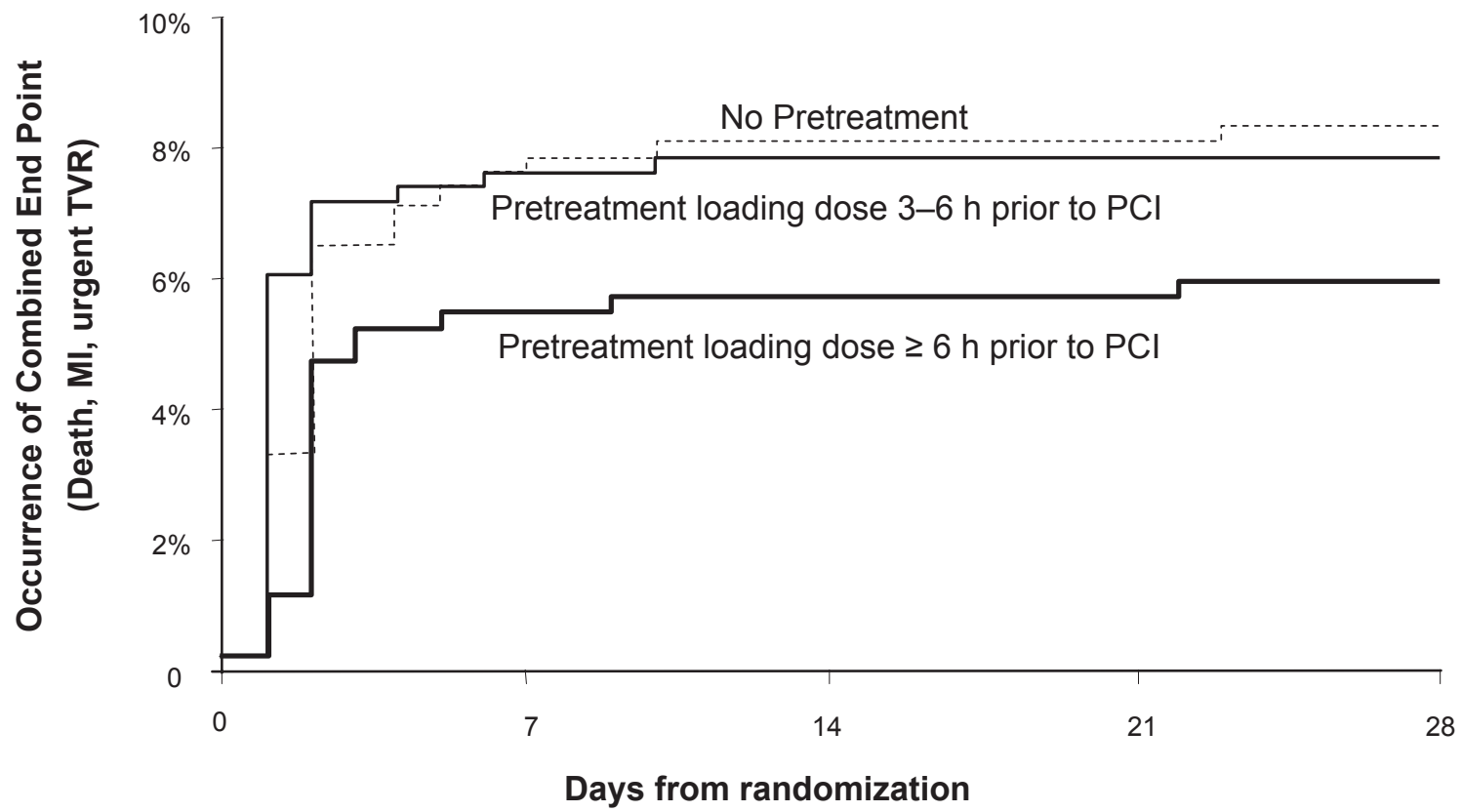

Figure 2 CREDO combined end points at 28 Days. Reproduced with permission from Steinhubl et al.JAMA. 2002;288(I9):24I I-2420. ${ }^{19}$ Copyright @ 2005 American Medical Association. All rights reserved.

significance $(\mathrm{p}=0.051$, Figure 2$)$. In a subsequent analysis of these data, the CREDO investigators found that the benef it of clopidogrel loading became highly significant when administered at least 15 hours prior the procedure. ${ }^{22}$ Thus CREDO expanded the indication for clopidogrel therapy to a more stable ischemic population group and also suggested the optimal clopidogrel therapy prior to PCI in terms of reduction of periprocedural thrombotic events was a loading dose of
$300 \mathrm{mg}$ given at least 6 hours and optimally 15 hours prior to PCI followed by a maintenance daily dose.

PCI-CLARITY ${ }^{20}$ examined the outcomes of 1863 patients with STEMI who per study protocol had received fibrinolytic therapy followed by routine angiography and then underwent PCI at the discretion of the treating physician. These patients had been randomly assigned to clopidogrel $(\mathrm{N}=933)$ or placebo $(\mathrm{N}=930)$ prior to angiography. Patients randomized 
to clopidogrel received a $300 \mathrm{mg}$ loading dose followed by $75 \mathrm{mg}$ per day with the load being given within 45 minutes of the start of fibrinolytic therapy. The primary efficacy outcome was a composite of $\mathrm{CV}$ death, recurrent $\mathrm{MI}$, or stroke from the time of PCI to 30 days after randomization. CK-MB was measured 3 times over 24 hours following intervention to ensure systematic monitoring for post-PCI MI which was defined as CK-MB of at least 3 times ULN and if CK-MB was already elevated, a CK-MB increase by $50 \%$ over the previous value. The median number of days of clopidogrel administration prior to PCI was 3 days.

Pretreatment with clopidogrel significantly reduced the rate of CV death, MI, or stroke following PCI through 30 days after randomization $(3.6 \%$ versus $6.2 \%, \mathrm{p}=0.008)$. The benefit of pretreatment was directionally consistent among all the components of the composite end point. Event curves revealed the two treatment groups separated soon after PCI and continued to diverge over time (Figure 3). Even though the median duration of time between randomization and PCI was 3 days, the benefit of pretreatment seemed consistent across the broad range of pretreatment durations ranging from 6 hours to 8 days.

Taken together, the PCI-CURE, CREDO, and PCICLARITY studies demonstrated a consistent benefit with clopidogrel pretreatment in terms of reduction of shortterm cardiac events following PCI (Figure 4). As CREDO and PCI-CLARITY specifically examined and included periprocedural MI in their short term composite primary endpoint analysis, the use of clopidogrel certainly suggests a reduction in this event while analysis of PCI-CURE suggested a decrease in short-term events driven specifically by a decrease in periprocedural MIs.

The benefit of clopidogrel in PCI in terms of reduction of myocardial necrosis is demonstrated in reduced short term cardiac events but also can be inferred from the higher rates of TIMI 3 flow seen in patients who receive clopidogrel pretreatment. In PCI-CLARITY, a higher percentage of patients receiving clopidogrel versus placebo had TIMI 3 flow at the time of their protocol mandated angiography. ${ }^{20} \mathrm{~A}$ meta-analysis of 38 trials comparing patients with STEMI treated with PCI receiving clopidogrel pretreatment versus placebo found similar results with a highly significant increase in TIMI 3 flow in patients pretreated with clopidogrel (OR 1.53, $\mathrm{p}<0.0001){ }^{23}$ This meta-analysis also confirmed the significant decrease in short term ( $<42$ days) mortality and death/reinfarction seen PCI-CURE, CREDO, and PCI-CLARITY when patients were pretreated with clopidogrel (Figure 5).

These studies demonstrate the importance of clopidogrel pretreatment in terms of reducing periprocedural myonecrosis and associated short term cardiac events and is the basis for the current American College of Cardiology (ACC)/ American Heart Association (AHA) class 1 recommendation for the use of clopidogrel pretreatment with a $300 \mathrm{mg}$ loading dose given at least 6 hours before PCI in patients

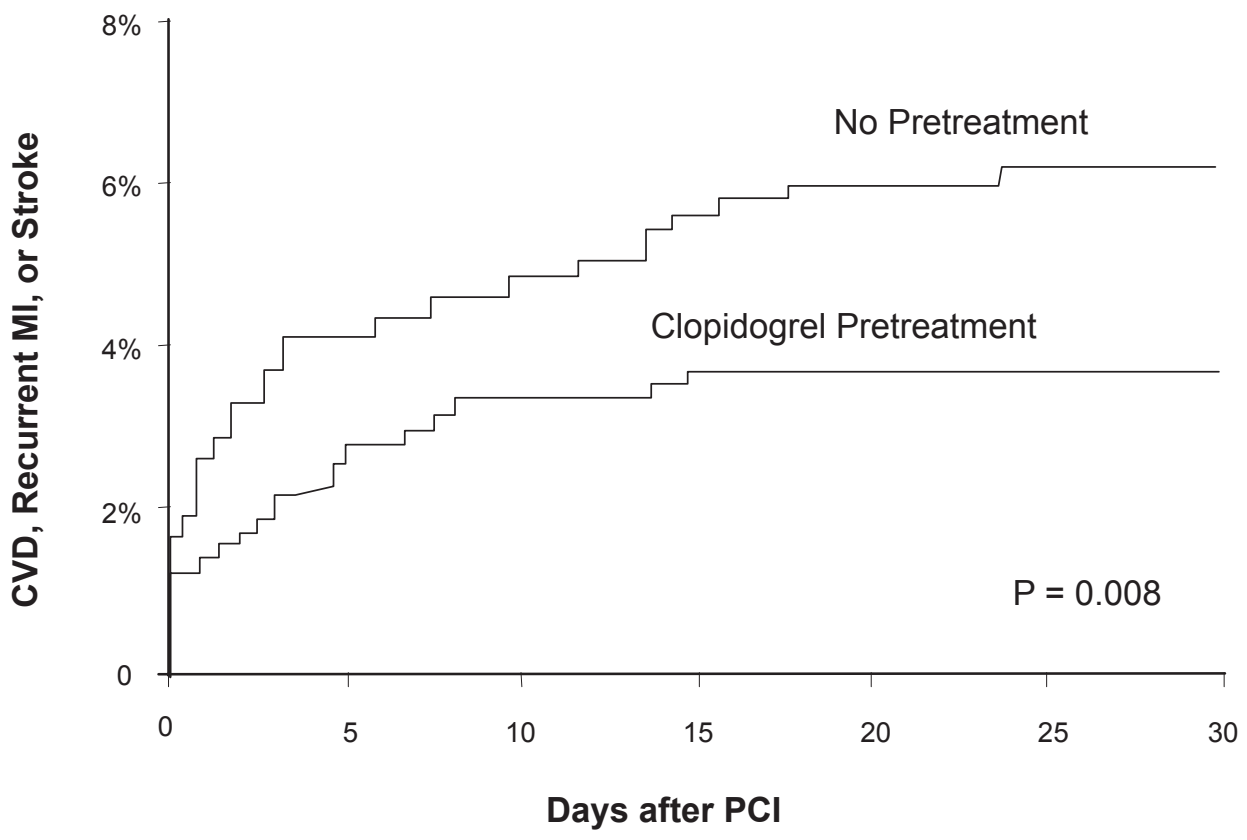

Figure 3 Outcomes at 30 days in the PCI-CLARITY study. Reproduced with permission from Sabatine et al. JAMA. 2005;294(I0):I224-I232.20 Copyright (C) 2005 American Medical Association. All rights reserved. 


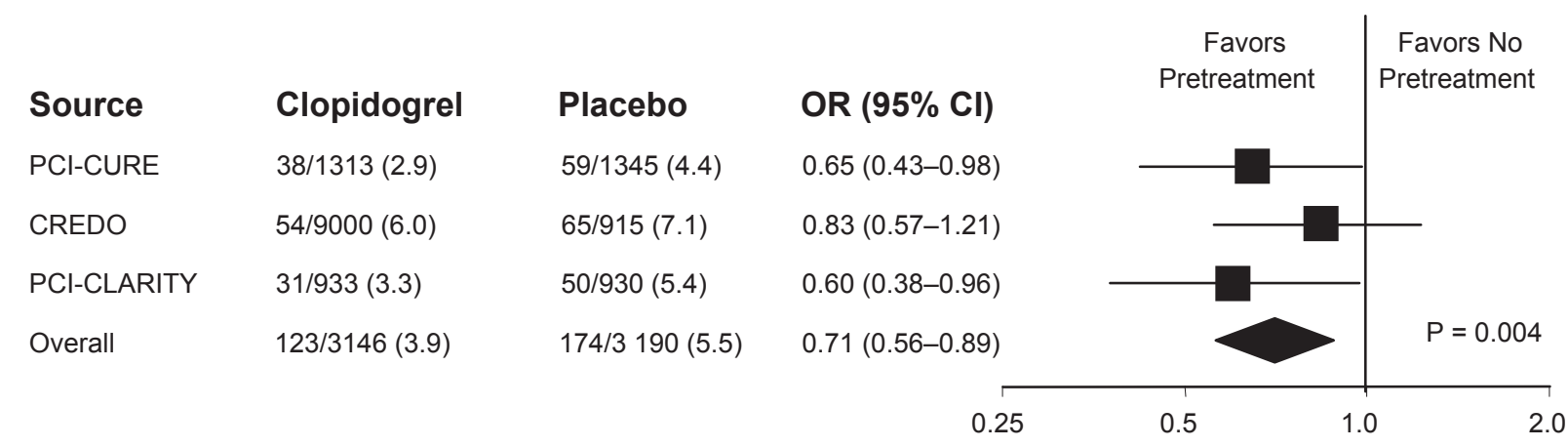

Figure 4 Summary of $\mathrm{PCl}$ studies assessing the effect of clopidogrel treatment. Reproduced with permission from Sabatine et al.JAMA. 2005;294(I0): I224-I232.20 Copyright $\left({ }^{\circ}\right.$ 2005 American Medical Association. All rights reserved.

with acute coronary syndrome as well as in those undergoing elective intervention. ${ }^{24}$

\section{Optimal pre-treatment dose: higher loading dose and chronic therapy}

Observational data demonstrated that a $600 \mathrm{mg}$ loading dose of clopidogrel caused an earlier and stronger inhibition of adenosine diphosphate (ADP)-induced platelet activation than a $300 \mathrm{mg}$ loading dose. ${ }^{25,26}$ Based on these studies, the Antiplatelet Therapy for Reduction of Myocardial Damage during Angioplasty (ARMYDA-2) study was carried out to test the hypothesis of whether a higher loading dose of $600 \mathrm{mg}$ clopidogrel would reduce post-PCI events compared to a standard $300 \mathrm{mg}$ dose in patients undergoing elective PCI. ${ }^{27}$ A total of 329 patients scheduled for PCI were randomized to a $600 \mathrm{mg}(\mathrm{N}=163)$ or $300 \mathrm{mg}(\mathrm{N}=166)$ loading regimen of clopidogrel given 4 to 8 hours (mean of 6 hours) before the procedure. The primary endpoint of the study was 30-day occurrence of death, MI, or TVR. CK-MB, troponin I, and myoglobin levels were measured at baseline and at 8 and 24 hours after PCI and the secondary end points included any post-procedural increase of these markers above the ULN.
The primary endpoint occurred in $4 \%$ of patients in the high loading dose versus $12 \%$ in the standard loading group $(p=0.041)$. This reduction in the primary endpoint was driven entirely by a decrease in rates of periprocedural MI; $15 \mathrm{MI}$ occurred in the $300 \mathrm{mg}$ group versus 5 in the $600 \mathrm{mg}$ group. Peak values of all cardiac biomarkers were significantly lower in patients treated with the $600 \mathrm{mg}$ regimen (Figure 6).

The ARMYDA-2 study suggested patients given a $600 \mathrm{mg}$ loading dose of clopidogrel 6 hours prior to PCI experienced fewer periprocedural MI than those receiving a $300 \mathrm{mg}$ loading dose. The majority of patients in both arms of the ARMYDA-2 study were low risk stable angina patients ( $75 \%$ in both high and conventional loading dose arms). When the higher loading $600 \mathrm{mg}$ dose was tested exclusively in higher risk patients with NSTE-ACS, similar superior outcomes at 1 month post-PCI was noted in the high-risk patients given $600 \mathrm{mg}$ loading dose versus conventional $300 \mathrm{mg}$ dose; a 1-month follow up period revealed recurrent cardiovascular events occurring at $5 \%$ and $12 \%$ respectively $(\mathrm{p}=0.02) .{ }^{28}$ A meta-analysis conducted by Lotrionte et $\mathrm{l}^{29}$ further investigated the benefit of high dose clopidogrel loading versus standard $300 \mathrm{mg}$

\begin{tabular}{lccc}
\hline & \multicolumn{2}{l}{ Unadjusted treatment effect } \\
& OR & $\mathbf{9 5 \% ~ C l}$ & P \\
\hline TIMl grade 2/3 flow & 1.53 & $1.39-1.68$ & $<0.0001$ \\
Mortality & 0.52 & $0.41-0.67$ & $<0.0001$ \\
Death/reinfarction & 0.50 & $0.40-0.62$ & $<0.0001$ \\
\hline
\end{tabular}

Figure 5 Meta-analysis of clopidogrel pretreatment in STEMI patients undergoing PCl with respect to short-term outcomes. Reproduced with permission from Vlaar PJ, Svilaas T, Damman K, et al. Impact of pretreatment with clopidogrel on initial patency and outcome in patients treated with primary percutaneous coronary intervention for ST-segment elevation myocardial infarction: a systematic review. Circulation. 2008; I 18(18): 1828-1836. ${ }^{23}$ Copyright (C) 2008 Lippincott Williams and Wilkins. 


\section{$600 \mathrm{mg} \square 300 \mathrm{mg}$ \\ Loading Dose}

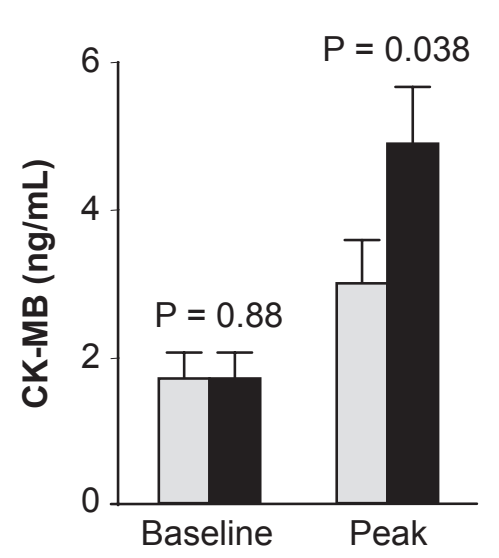

$P=0.021$

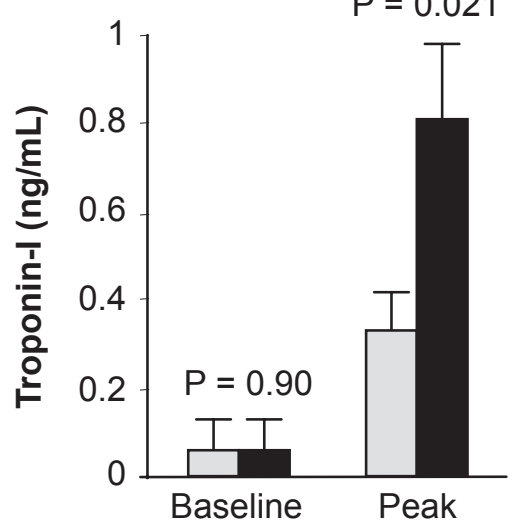

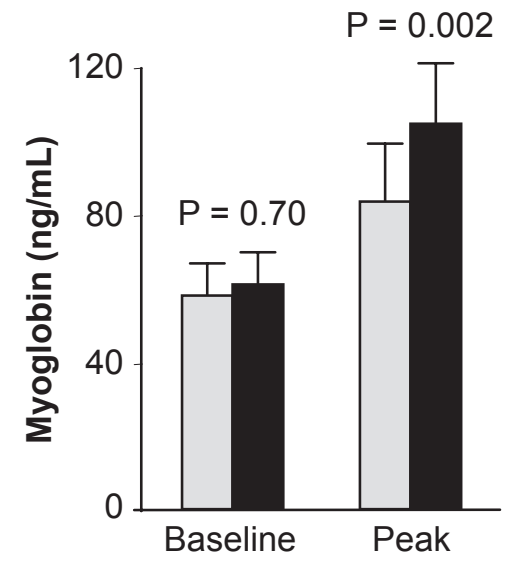

Figure 6 Peri-procedural biomarker changes in the ARMYDA-2 study. Reproduced with permission from Patti G, Colonna G, Pasceri V, et al. Randomized trial of high loading dose of clopidogrel for reduction of periprocedural myocardial infarction in patients undergoing coronary intervention: results from the ARMYDA-2 (Antiplatelet therapy for Reduction of MYocardial Damage during Angioplasty) study. Circulation. 2005; I I ( I6):2099-2106. ${ }^{27}$ Copyright (C) 2005 Lippincott Williams and Wilkins.

loading in patients undergoing PCI. The meta-analysis included 10 trials (7 randomized and 3 non-randomized) with a total of 1567 patients enrolled. Overall, a high loading dose $(>300 \mathrm{mg}$ ) proved significantly superior to a standard loading dose in terms of reducing early cardiac death or non-fatal MI post PCI (odds ratio 0.54, $\mathrm{p}=0.02$ ). This analysis also found that patients at highest risk, defined by the presence of elevated cardiac biomarkers, benefited the most with the increased loading dose.

Although these studies suggest the beneficial role for a $600 \mathrm{mg}$ clopidogrel loading dose versus the $300 \mathrm{mg}$ loading dose, the optimal timing of the higher dose is somewhat controversial. In the ARMYDA-2 trial, the $600 \mathrm{mg}$ loading dose was given between $4-8$ hours with a mean of 6 hours prior to PCI suggesting the optimal timing is 6 hours prior to intervention. Platelet functional studies have shown that the $600 \mathrm{mg}$ dose of clopidogrel achieves maximal platelet inhibition 2 hours after administration. ${ }^{30}$ This has led to the overall consensus that the $600 \mathrm{mg}$ loading dose should be given at least 2 hours, and optimally 6 hours, prior to PCI.

The Intracoronary Stenting and Antithrombotic Regimen: Choose Between 3 High Oral Doses for Immediate Clopidogrel Effect (ISAR-CHOICE) trial investigated whether loading doses higher than $600 \mathrm{mg}$ of clopidogrel exerted additional suppression of platelet function. ${ }^{31}$ The study compared the effects of 300,600, and $900 \mathrm{mg}$ clopidogrel doses on platelet function among stable patients scheduled for coronary angiography. Sixty patients were assigned to 1 of 3 clopidogrel loading schemes in a randomized, double blinded manner. Optical aggregometry was performed before and 4 hours after administration of clopidogrel. At 4 hours, patients treated with both 600 and $900 \mathrm{mg}$ doses demonstrated greater inhibition of platelet aggregation (IPA) than the $300 \mathrm{mg}$ dose. No significant difference in platelet inhibition was found between the 600 and $900 \mathrm{mg}$ doses. On the basis of this finding, the ISAR-CHOICE trial concluded that single clopidogrel loading dosages $>600 \mathrm{mg}$ was not associated with any additional suppression of platelet activity. The trial also found, through the measured levels of clopidogrel metabolites in the differing loading dose schemes, that the maximal clopidogrel loading dose of $600 \mathrm{mg}$ was perhaps related to impaired intestinal absorption beyond the $600 \mathrm{mg}$ dose. ${ }^{31}$

The Assessment of the Best Loading Dose of Clopidogrel to Blunt Platelet Activation, Inflammation, and Ongoing Necrosis (ALBION) trial, in contrast to ISAR-CHOICE found that there was greater platelet inhibition with $900 \mathrm{mg}$ loading dose of clopidogrel compared to $600 \mathrm{mg}$ loading dose in reference to the $300 \mathrm{mg}$ baseline loading dose. ${ }^{32}$ The ALBION study assessed platelet aggregation through standard ADP induced optical aggregometry in 35 patients randomized to $300 \mathrm{mg}, 34$ patients randomized to $600 \mathrm{mg}$, and 34 patients randomized to $900 \mathrm{mg}$ clopidogrel. Platelet aggregometry was measured 8 times in the first 6 hours and the study found increased inhibition of platelet activity (IPA) at higher loading dosage compared to standard dose with near equal time to maximal effect at 5 to 6 hours. It should be noted that no direct comparison of degree of platelet inhibition was made between the $600 \mathrm{mg}$ and $900 \mathrm{mg}$ doses. The trial was too small to assess clinical outcomes but did suggest 
that increased loading dosages may be useful in higher risk patients as there was increased platelet inhibition.

A related question of clopidogrel dosing involves whether patients who are already receiving a maintenance dose of clopidogrel should receive a booster load. The ARMYDA-4 study examined the efficacy of a reloading dose of clopidogrel in patients with stable angina or NSTE-ACS undergoing PCI who were on chronic clopidogrel therapy. ${ }^{33}$ Investigators followed patients randomly assigned to a reload arm $(\mathrm{N}=180$ ) with a $600 \mathrm{mg}$ clopidogrel load given 4 to 8 hours prior to PCI versus patients randomly assigned to a placebo $\operatorname{arm}(\mathrm{N}=180)$. The primary endpoint was a composite of death, MI, and TVR at 30 days. The secondary endpoints included increase in post-procedure biomarkers, peak values of myocardial enzymes, point of care evaluation of platelet activity, and bleeding complications. The composite primary endpoint was identical in the reload and placebo arms (8\% versus $7 \%$ ). The primary endpoint was driven exclusively by MI as there were no deaths and no TVR. Myocardial markers, platelet aggregometry, and bleeding complications were also identical in both arms. This study concluded that patients on chronic clopidogrel did not require reloading prior to PCI.

The main limitations of the ARMYDA-4 study included its small size and the fact that the majority of the enrolled patients were relatively stable with less than one third of the patients presenting with ACS. Subsequently, the ARMYDARELOAD study examined a higher risk population of patients taking chronic clopidogrel therapy. ${ }^{33}$ The study followed 436 patients on chronic clopidogrel therapy (at least 10 days) of which 167 patients had presented with ACS and examined the effect of reloading with $600 \mathrm{mg}$ clopidogrel versus placebo on post-PCI outcomes. The primary endpoint was a composite endpoint of death, MI, and TVR at 30 days. Platelet reactivity was also measured in both groups. Overall, there was again no demonstrable difference in the primary endpoint between the 2 groups (7\% clopidogrel reload arm versus 9\% in placebo arm, $p=0.7$ ) but when the ACS subgroup was analyzed alone, there was a significant reduction in the primary endpoint in the reload arm (7\% versus $18 \%, \mathrm{p}=0.035)$. Platelet reactivity was also significantly lower in the ACS subgroup receiving a $600 \mathrm{mg}$ reload. Thus although this study was essentially negative, it did suggest the beneficial role of a $600 \mathrm{mg}$ reload given 4 to 8 hours prior to PCI in patients on chronic clopidogrel therapy presenting with ACS.

In the Dose Effect of Clopidogrel Reloading in Patients Already on 75 mg Maintenance Dose: the Reload With Clopidogrel Before Angioplasty in Subjects Treated Long Term
With Dual Antiplatelet Therapy (RELOAD) Study, Collet et al examined whether patients on chronic clopidogrel benefited from a $900 \mathrm{mg}$ reload dose. ${ }^{34}$ The dosage scheme of reloading was given in 3 different strategies allowing further examination of the hypothesis raised by ISAR-CHOICE that there was a maximal absorption threshold of $600 \mathrm{mg}$ which limited the benefit of higher load dosing schemes. The study's primary endpoint was inhibition of platelet activity as measured by optical aggregometry. Clinical endpoints of death, MI, and TVR were also measured at 30 days. The study found that clopidogrel reloading with $900 \mathrm{mg}$ did have significant increase in platelet inhibition compared to lower loading doses and there was no evidence of an absorption threshold which limited the benefit of the $900 \mathrm{mg}$ loading dose. There was no difference in the clinical endpoint between any of the dosing schemes. Thus although the study provided evidence for the use of a $900 \mathrm{mg}$ loading dose in terms of platelet reactivity, the lack of clinical benefit made the findings difficult to interpret.

Overall, all of the above studies have suggested that a clopidogrel loading dose of $600 \mathrm{mg}$ or $900 \mathrm{mg}$ may be more beneficial than a $300 \mathrm{mg}$ loading dose in terms of reducing postPCI events. As most of these trials had small sample sizes, ACC/ AHA guidelines still officially considers clopidogrel $300 \mathrm{mg}$ as an adequate loading dose but it does note that higher loading dose can be considered prior to PCI. ${ }^{24}$ The ongoing Clopidogrel Optimal Loading Dose Usage to Reduce Recurrent Events/ Optimal Antiplatelet Strategy for Interventions (OASIS-7) trial will randomize 14,000 high risk NSTE-ACS patients to $300 \mathrm{mg}$ versus $600 \mathrm{mg}$ loading clopidogrel dose prior to PCI and should help further clarify the benefit of higher loading doses in terms of short-term $\left(<30\right.$ days) post-PCI events. ${ }^{35}$

\section{Personalized therapy}

Much of the impetus for the higher dosing of clopidogrel is due to the wide variability of clopidogrel associated platelet inhibition, ie, the presence of clopidogrel resistance and its clinical implications. Hochholzer et al showed that in patients undergoing elective PCI who were pre-loaded with $600 \mathrm{mg}$ clopidogrel, the degree of platelet inhibition as measured by ADP-induced optical aggregometry prior to intervention predicted the likelihood of post-PCI myocardial events. ${ }^{36}$ Patients within the lower third and fourth quartile of platelet inhibition were at a significantly higher risk of worse shortterm outcomes compared to those in the higher quartiles of platelet inhibition (Figure 7). This finding was also seen in the Antiplatelet Therapy for the Reduction of Myocardial Damage during Angioplasty - Platelet Reactivity Predicts Outcomes (ARMYDA-PRO) study which also assessed 


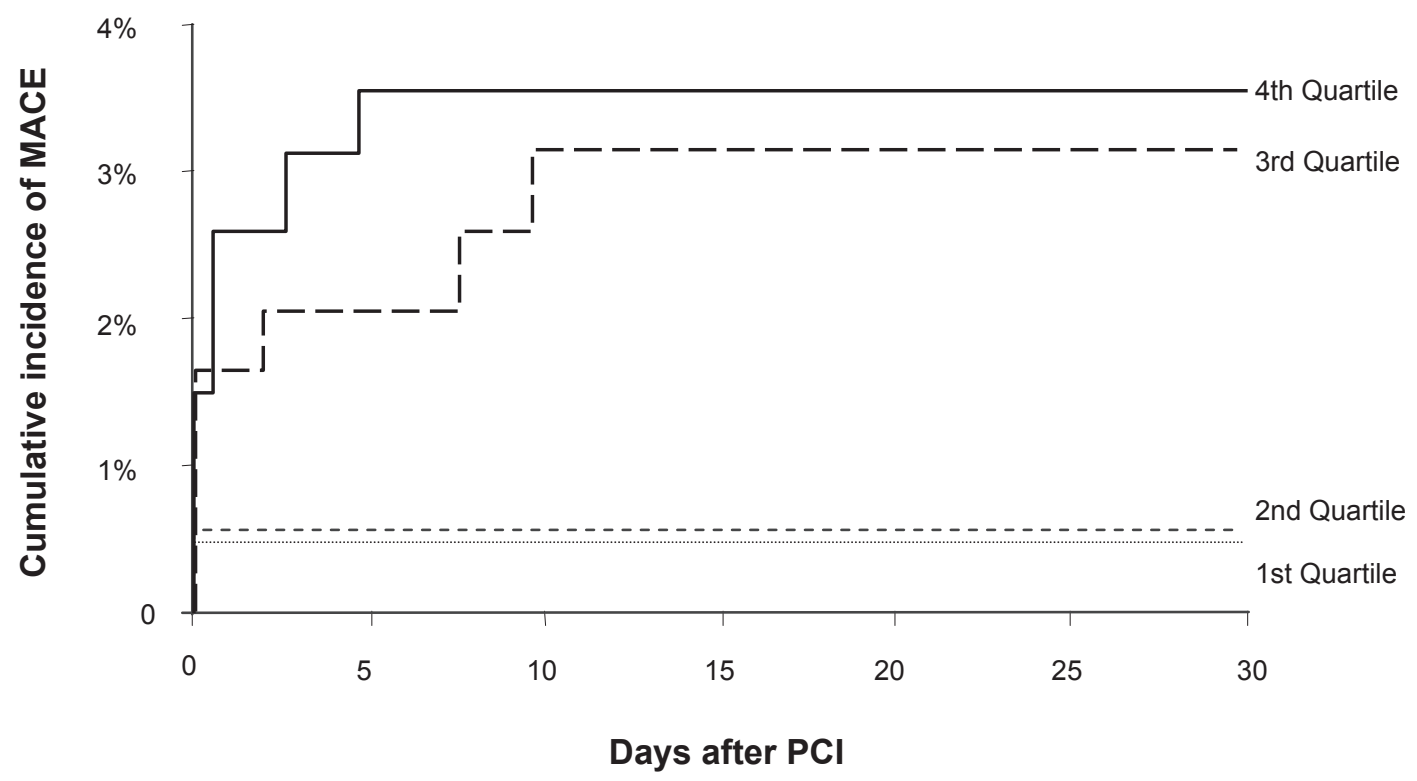

Figure 7 Degree of platelet inhibition and major cardiovascular events. Reproduced with permission from HochholzerW, Trenk D, Bestehorn HP, et al. Impact of the degree of peri-interventional platelet inhibition after loading with clopidogrel on early clinical outcome of elective coronary stent placement. J Am Coll Cardiol. 2006;48(9): 1742-1750.36 Copyright (c) 2006 Elsevier.

platelet activity after a $600 \mathrm{mg}$ loading clopidogrel dose but used a rapid cartridge based assay (VerifyNow P2Y12 Assay) rather than optical aggregometry. ${ }^{37}$

These studies led to the hypothesis that tailored therapy to overcome clopidogrel resistance could result in improved post-PCI outcomes. Although ADP-induced optical aggregometry is considered the gold standard in terms of assessing platelet function in patients receiving clopidogrel, it is a difficult test to perform at the bedside therefore its use in tailored therapy is limited. ${ }^{38}$ Additionally, ADP-induced optical aggregometry is somewhat non-specific as the effects of ADP on the P2Y1 receptor are included in this test but clopidogrel action does not involve this receptor. Schwarz et al found that the activity of clopidogrel and ticlopidine with its associated blockade of the P2Y12 receptor could be monitored through analysis of the intracellular vasodilatorstimulated phosphoprotein (VASP) phosphorylation state..$^{39}$ Barragan et al used flow cytometry to quantitate the VASP phosphorylation state and developed the VASP index which is essentially a ratio of platelet activity that is inversely related to the effect of clopidogrel..$^{40}$ This study also found that a VASP index greater than $50 \%$ was associated with a higher risk of subacute stent thrombosis.

Bonello et al prospectively examined the use of the tailored clopidogrel dosing using the VASP index on post-PCI outcomes. ${ }^{41}$ This study initially measured the VASP index 24 hours after a $600 \mathrm{mg}$ clopidogrel loading dose in patients undergoing planned PCI for refractory angina, silent ischemia on stress testing, and NSTEMI. Patients with a VASP index $>50 \%$ were officially enrolled in the study $(\mathrm{N}=162)$ and were randomized to a control group $(\mathrm{N}=84)$ versus further VASP-guided clopidogrel dosing $(\mathrm{N}=78)$. In the control group, PCI was carried out without additional clopidogrel. In the VASP-guided group, patients were given up to 3 additional $600 \mathrm{mg}$ clopidogrel doses in 24-hour increments until the VASP index was below 50\%. The effect of each additional clopidogrel dose is shown in Figure 8. One-month follow-up demonstrated a significant improvement in clinical outcomes with the control group having an overall major adverse cardiovascular event (MACE) rate of $10 \%$ including 1 case of acute stent thrombosis and 3 cases of subacute stent thrombosis versus $0 \%$ in the VASP guided group $(\mathrm{p}=0.007$, Figure 9). This study provided clinical evidence for tailored clopidogrel therapy based on platelet activity but is limited by its small sample size. The ongoing Gauging Responsiveness With A VerifyNow Assay-Impact On Thrombosis And Safety (GRAVITAS) trial will compare outcomes in 6600 patients randomized to tailored therapy using platelet functional testing versus standard therapy and should help clarify whether there is real benefit to this approach.

\section{Benefit of clopidogrel and adjunctive therapies}

All studies demonstrating the benefit of clopidogrel in terms of reducing post-PCI myocardial necrosis have been against the background of aspirin use so dual antiplatelet therapy is 


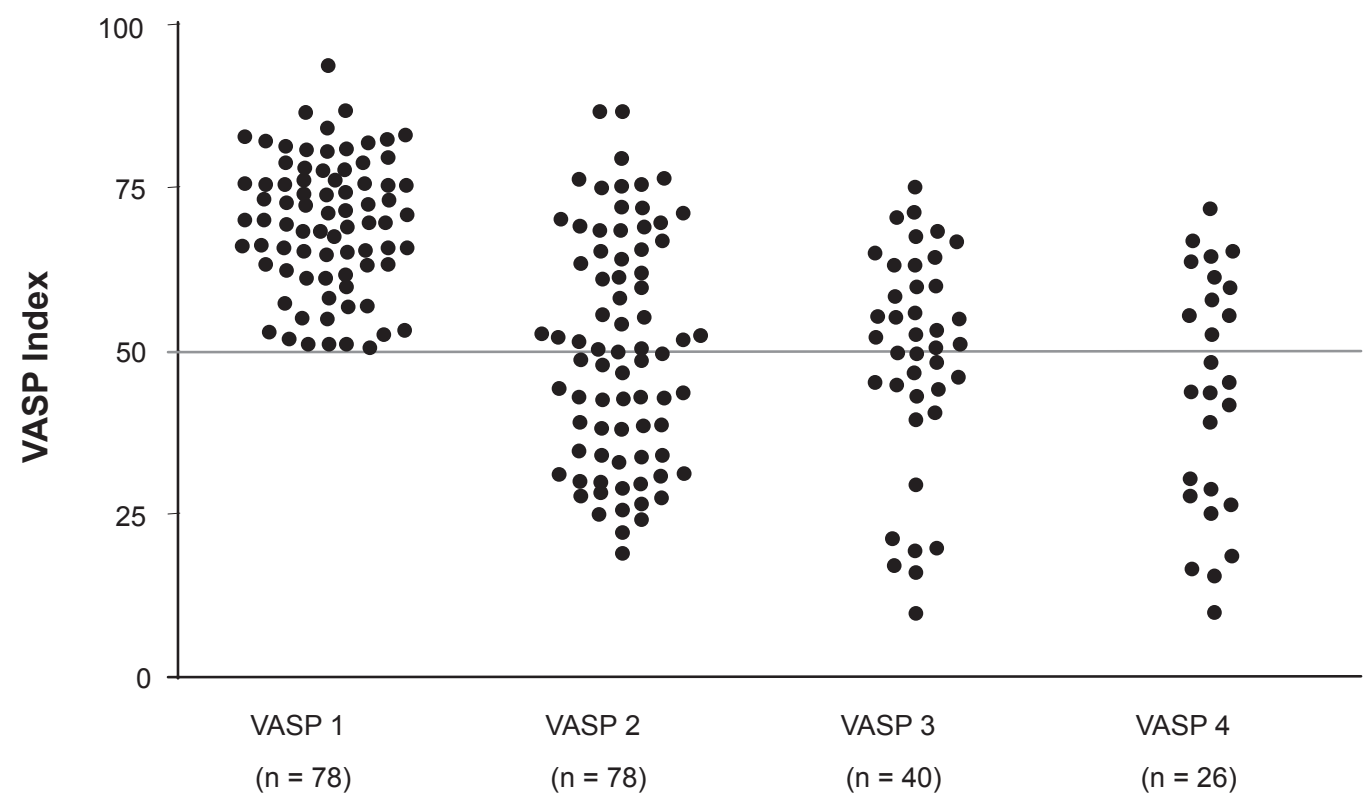

Figure 8 Effect of additional clopidogrel dosing on VASP Index. Reproduced with permission from Bonello L, Camoin-Jau L,Arques S, et al.Adjusted clopidogrel loading doses according to vasodilator-stimulated phosphoprotein phosphorylation index decrease rate of major adverse cardiovascular events in patients with clopidogrel resistance: a multicenter randomized prospective study. J Am Coll Cardiol. 2008;5I(I4):I404-I4II. ${ }^{1 \mid}$ Copyright (c) 2008 Elsevier.

mandatory for optimal benefit. Additionally antithrombotic use with heparin or enoxaparin was also routine in all studies demonstrating the benefits of clopidogrel in the setting of PCI. A meta-analysis by Sabatine et $\mathrm{al}^{42}$ examined the PCI-CURE, CREDO, and PCI-CLARITY trials and demonstrated that there was added benefit to pretreatment with clopidogrel regardless of the use glycoprotein IIB/IIIA inhibitors (GP IIB/IIIA inhibitors). This suggested that patients undergoing PCI who were started on GP IIB/IIIA inhibitors still benefited from the presence of clopidogrel loading in terms of reducing post-PCI events provided the dose was given adequately before the actual time of PCI. The Intracoronary Stenting and Anti-thrombotic Regimen: Rapid
Early Action for Coronary Treatment (ISAR-REACT) trial demonstrated that in low risk patients, there is in fact no benefit, in terms of short term clinical post-PCI events, to adding GP IIB/IIIA inhibitors if clopidogrel loading with $600 \mathrm{mg}$ is administered at least 2 hours prior to PCI. Thus in low risk patients, clopidogrel loading with $600 \mathrm{mg}$ seems to provide adequate platelet activity inhibition with no need for further anti-platelet therapy. ${ }^{43}$ In contrast higher risks patients, as defined by the presence of positive troponins, do seem to have additional benefit in terms of reduced post-PCI events when a GP IIB/IIIA inhibitor is used in addition to clopidogrel pretreatment. This was demonstrated by the ISARREACT 2 trial which enrolled 2022 patients presenting with

\begin{tabular}{llc}
\hline End point & $\begin{array}{l}\text { Control group } \\
\mathbf{n}(\%)\end{array}$ & $\begin{array}{l}\text { VASP-Guided group } \\
\mathbf{n}(\%)\end{array}$ \\
\hline Cardiovascular death & $2(2)$ & 0 \\
Acute stent thrombosis & $1(1)$ & 0 \\
Subacute stent thrombosis & $3(4)$ & 0 \\
$\begin{array}{l}\text { Recurrent acute coronary } \\
\text { syndrome }\end{array}$ & $2(2)$ & 0 \\
All MACE & $8(10)$ & 0 \\
\hline
\end{tabular}

Figure 9 One month clinical outcomes standard versus VASP-guided clopidogrel loading. Reproduced with permission from Bonello L, Camoin-Jau L,Arques S, et al. Adjusted clopidogrel loading doses according to vasodilator-stimulated phosphoprotein phosphorylation index decrease rate of major adverse cardiovascular events in patients with clopidogrel resistance: a multicenter randomized prospective study. J Am Coll Cardiol. 2008;5 (14):1404-1411.41 Copyright @ 2008 Elsevier. 
NSTE-ACS undergoing PCI after being loaded with $600 \mathrm{mg}$ clopidogrel at least 2 hours prior to intervention. ${ }^{44}$ Patients were administered abciximab $(\mathrm{N}=1012)$ versus placebo ( $\mathrm{N}=1010)$ in a double-blinded, randomized manner at the time of PCI. A composite of ischemic events (death, MI, urgent TVR) was assessed 30 days post PCI and found to be significantly reduced in the abciximab group versus placebo ( $8.9 \%$ versus $11.9 \%, p=0.03)$. This benefit was limited only to patients with an elevated troponin. The ACUITY trial demonstrated that the use of the direct thrombin inhibitor bivalirudin was not inferior to the use of GP IIB/IIIA inhibitor and enoxaparin or unfractionated heparin (UFH) in the management of patients presenting with NSTE-ACS with respect to 30-day rates of a composite endpoint of death, MI, or urgent TVR..$^{45}$ Clopidogrel therapy was not mandated by protocol in either arm but a loading dose of $300 \mathrm{mg}$ no later than 2 hours post-PCI and daily maintenance therapy of $75 \mathrm{mg}$ was recommended. There was no significant difference in the proportion of patients receiving clopidogrel therapy in the bivalirudin versus GP IIB/IIIA plus enoxaparin or UFH arms (64.2\% versus $62.3 \%$ respectively). Subgroup analysis of this study suggested that patients on bivalirudin monotherapy who did not receive clopidogrel prior to PCI had increased ischemic events post-procedure compared to patients receiving GP IIB/IIIA and enoxaparin or UFH (9.1\% versus 7.1\%; 95\% CI 1.03 to 1.63$)$. This suggested that use of bivalirudin in patients with NSTE-ACS requiring PCI should be limited to patients who have been pre-treated with clopidogrel. A subsequent post-hoc analysis by Lincoff et $\mathrm{al}^{46}$ found that as long as clopidogrel is administered before or within 30 minutes post-PCI, treatment with bivalirudin is equivalent (non-inferior) to enoxaparin or UFH and GP IIB/IIIA inhibitor in terms of short-term post-PCI ischemic outcomes.

\section{Conclusion}

Clopidogrel is an important pharmacologic agent used to reduce MI post-PCI as assessed directly by the evaluation of cardiac biomarkers post-intervention and indirectly by the evaluation of short term ischemic events. The optimal dose of clopidogrel is considered to be at least $300 \mathrm{mg}$ given at least 6 to 15 hours prior to PCI but there is considerable evidence to suggest a loading dose of $600 \mathrm{mg}$ given 2 to 6 hours prior to PCI may be more efficacious in limiting post-PCI events. Doses higher than $600 \mathrm{mg}$ may be still more efficacious especially in patients with evidence of clopidogrel resistance as assessed by platelet function testing but this is controversial and requires further randomized testing to verify. The benefit of clopidogrel therapy in the reduction of post-PCI ischemic events is independent and complementary to other adjunctive therapies including aspirin, UFH/enoxaparin, GP IIB/IIIA, and bivalirudin.

\section{Disclosures}

The authors have no conflicts of interest to disclose.

\section{References}

1. Sheldon WC. Trends in cardiac catheterization laboratories in the United States. Catheter Cardiovasc Interv. 2001;53(1):40-45.

2. Bhatt DL, Topol EJ. Does creatinine kinase-MB elevation after percutaneous coronary intervention predict outcomes in 2005? Periprocedural cardiac enzyme elevation predicts adverse outcomes. Circulation. 2005;112(6):906-915; discussion 923.

3. Thygesen K, Alpert JS, White HD. Universal definition of myocardial infarction. J Am Coll Cardiol. 2007;50(22):2173-2195.

4. Brener SJ, Lytle BW, Schneider JP, et al. Association between CK-MB elevation after percutaneous or surgical revascularization and three-year mortality. J Am Coll Cardiol. 2002;40(11):1961-1967.

5. Mukherjee D, Oz M, Prager R, et al. Elective coronary revascularization, an iatrogenic form of acute coronary syndrome: how can clinicians reduce the risks? Am Heart J. 2004;148(3):371-377.

6. Dangas G, Mehran R, Feldman D, et al. Postprocedure creatine kinase-MB elevation and baseline left ventricular dysfunction predict one-year mortality after percutaneous coronary intervention. Am J Cardiol. 2002;89(5):586-589.

7. Kugelmass AD, Cohen DJ, Moscucci M, et al. Elevation of the creatine kinase myocardial isoform following otherwise successful directional coronary atherectomy and stenting. Am J Cardiol. 1994;74(8): 748-754.

8. Wiviott SD. Intensity of antiplatelet therapy in patients with acute coronary syndromes and percutaneous coronary intervention: the promise of prasugrel? Cardiol Clin. 2008;26(4):629-637.

9. Topol EJ. Toward a new frontier in myocardial reperfusion therapy: emerging platelet preeminence. Circulation. 1998;97(2):211-218.

10. Coller BS, Shattil SJ. The GPIIb/IIIa (integrin alphaIIbbeta3) odyssey: a technology-driven saga of a receptor with twists, turns, and even a bend. Blood. 2008;112(8):3011-3025.

11. Wiviott SD, Braunwald E, Angiolillo DJ, et al. Greater clinical benefit of more intensive oral antiplatelet therapy with prasugrel in patients with diabetes mellitus in the trial to assess improvement in therapeutic outcomes by optimizing platelet inhibition with prasugrelThrombolysis in Myocardial Infarction 38. Circulation. 2008;118(16): 1626-1636.

12. Schomig A, Neumann FJ, Kastrati A, et al. A randomized comparison of antiplatelet and anticoagulant therapy after the placement of coronaryartery stents. N Engl J Med. 1996;334(17):1084-1089.

13. Bertrand ME, Legrand V, Boland J, et al. Randomized multicenter comparison of conventional anticoagulation versus antiplatelet therapy in unplanned and elective coronary stenting. The full anticoagulation versus aspirin and ticlopidine (fantastic) study. Circulation. 1998;98(16): 1597-1603.

14. Cutlip DE, Leon MB, Ho KK, et al. Acute and nine-month clinical outcomes after "suboptimal" coronary stenting: results from the STent Anti-thrombotic Regimen Study (STARS) registry. J Am Coll Cardiol. 1999;34(3):698-706.

15. Jakubowski JA, Winters KJ, Naganuma H, et al. Prasugrel: a novel thienopyridine antiplatelet agent. A review of preclinical and clinical studies and the mechanistic basis for its distinct antiplatelet profile. Cardiovasc Drug Rev. 2007;25(4):357-374.

16. Michelson AD. P2Y12 antagonism: promises and challenges Arterioscler Thromb Vasc Biol. 2008;28(3):s33-s38. 
17. Bertrand ME, Rupprecht HJ, Urban P, et al. Double-blind study of the safety of clopidogrel with and without a loading dose in combination with aspirin compared with ticlopidine in combination with aspirin after coronary stenting: the clopidogrel aspirin stent international cooperative study (CLASSICS). Circulation. 2000;102(6):624-629.

18. Mehta SR, Yusuf S, Peters RJ, et al. Effects of pretreatment with clopidogrel and aspirin followed by long-term therapy in patients undergoing percutaneous coronary intervention: the PCI-CURE study. Lancet. 2001;358(9281):527-533.

19. Steinhubl SR, Berger PB, Mann JT, 3rd, et al. Early and sustained dual oral antiplatelet therapy following percutaneous coronary intervention: a randomized controlled trial. JAMA. 2002;288(19):2411-2420.

20. Sabatine MS, Cannon CP, Gibson CM, et al. Effect of clopidogrel pretreatment before percutaneous coronary intervention in patients with ST-elevation myocardial infarction treated with fibrinolytics: the PCI-CLARITY study. JAMA. 2005;294(10):1224-1232.

21. Mehta SR, Yusuf S. The Clopidogrel in Unstable angina to prevent Recurrent Events (CURE) trial programme; rationale, design and baseline characteristics including a meta-analysis of the effects of thienopyridines in vascular disease. Eur Heart J. 2000;21(24):2033-2041.

22. Steinhubl SR, Berger PB, Brennan DM, et al. Optimal timing for the initiation of pre-treatment with $300 \mathrm{mg}$ clopidogrel before percutaneous coronary intervention. J Am Coll Cardiol. 2006;47(5):939-943.

23. Vlaar PJ, Svilaas T, Damman K, et al. Impact of pretreatment with clopidogrel on initial patency and outcome in patients treated with primary percutaneous coronary intervention for ST-segment elevation myocardial infarction: a systematic review. Circulation. 2008;118(18):1828-1836.

24. Anderson JL, Adams CD, Antman EM, et al. ACC/AHA 2007 guidelines for the management of patients with unstable angina/non-ST-Elevation myocardial infarction: a report of the American College of Cardiology/ American Heart Association Task Force on Practice Guidelines (Writing Committee to Revise the 2002 Guidelines for the Management of Patients With Unstable Angina/Non-ST-Elevation Myocardial Infarction) developed in collaboration with the American College of Emergency Physicians, the Society for Cardiovascular Angiography and Interventions, and the Society of Thoracic Surgeons endorsed by the American Association of Cardiovascular and Pulmonary Rehabilitation and the Society for Academic Emergency Medicine. J Am Coll Cardiol. 2007;50(7):e1-e157.

25. Muller I, Seyfarth M, Rudiger S, et al. Effect of a high loading dose of clopidogrel on platelet function in patients undergoing coronary stent placement. Heart. 2001;85(1):92-93.

26. Gurbel PA, Bliden KP, Hayes KM, et al. The relation of dosing to clopidogrel responsiveness and the incidence of high post-treatment platelet aggregation in patients undergoing coronary stenting. $J \mathrm{Am}$ Coll Cardiol. 2005;45(9):1392-1396.

27. Patti G, Colonna G, Pasceri V, et al. Randomized trial of high loading dose of clopidogrel for reduction of periprocedural myocardial infarction in patients undergoing coronary intervention: results from the ARMYDA-2 (Antiplatelet therapy for Reduction of MYocardial Damage during Angioplasty) study. Circulation. 2005;111(16):2099-2106.

28. Cuisset T, Frere C, Quilici J, et al. Benefit of a 600-mg loading dose of clopidogrel on platelet reactivity and clinical outcomes in patients with non-ST-segment elevation acute coronary syndrome undergoing coronary stenting. J Am Coll Cardiol. 2006;48(7):1339-1345.

29. Lotrionte M, Biondi-Zoccai GG, Agostoni P, et al. Meta-analysis appraising high clopidogrel loading in patients undergoing percutaneous coronary intervention. Am J Cardiol. 2007;100(8):1199-1206.

30. Hochholzer W, Trenk D, Frundi D, et al. Time dependence of platelet inhibition after a 600-mg loading dose of clopidogrel in a large, unselected cohort of candidates for percutaneous coronary intervention. Circulation. 2005;111(20):2560-2564.

31. von Beckerath N, Taubert D, Pogatsa-Murray G, et al. Absorption, metabolization, and antiplatelet effects of 300-, 600-, and 900-mg loading doses of clopidogrel: results of the ISAR-CHOICE (Intracoronary Stenting and Antithrombotic Regimen: Choose Between 3 High Oral Doses for Immediate Clopidogrel Effect) Trial. Circulation. 2005;112(19):2946-2950.
32. Montalescot G, Sideris G, Meuleman C, et al. A randomized comparison of high clopidogrel loading doses in patients with non-ST-segment elevation acute coronary syndromes: the ALBION (Assessment of the Best Loading Dose of Clopidogrel to Blunt Platelet Activation, Inflammation and Ongoing Necrosis) trial. J Am Coll Cardiol. 2006;48(5):931-938.

33. di Sciascio G. The ARMYDA trials (Atorvastatin for Reduction of MYocardial Damage during Angioplasty) at Campus Bio-Medico University: rationale, results and future horizons. Fundam Clin Pharmacol. 2007;21 Suppl 2:41-43.

34. Collet JP, Silvain J, Landivier A, et al. Dose effect of clopidogrel reloading in patients already on 75-mg maintenance dose: the Reload with Clopidogrel Before Coronary Angioplasty in Subjects Treated Long Term with Dual Antiplatelet Therapy (RELOAD) study. Circulation. 2008;118(12):1225-1233.

35. Mehta SR, Bassand JP, Chrolavicius S, et al. Design and rationale of CURRENT-OASIS 7: a randomized, $2 \times 2$ factorial trial evaluating optimal dosing strategies for clopidogrel and aspirin in patients with ST and non-ST-elevation acute coronary syndromes managed with an early invasive strategy. Am Heart J. 2008;156(6):1080-1088 e1081.

36. Hochholzer W, Trenk D, Bestehorn HP, et al. Impact of the degree of peri-interventional platelet inhibition after loading with clopidogrel on early clinical outcome of elective coronary stent placement. J Am Coll Cardiol. 2006;48(9):1742-1750.

37. Patti G, Nusca A, Mangiacapra F, et al. Point-of-care measurement of clopidogrel responsiveness predicts clinical outcome in patients undergoing percutaneous coronary intervention results of the ARMYDA-PRO (Antiplatelet therapy for Reduction of MYocardial Damage during Angioplasty-Platelet Reactivity Predicts Outcome) study. J Am Coll Cardiol. 2008;52(14):1128-1133.

38. Michelson AD. Platelet function testing in cardiovascular diseases. Circulation. 2004;110(19):e489-e493.

39. Schwarz UR, Geiger J, Walter U, et al. Flow cytometry analysis of intracellular VASP phosphorylation for the assessment of activating and inhibitory signal transduction pathways in human platelets - definition and detection of ticlopidine/clopidogrel effects. Thromb Haemost. 1999;82(3):1145-1152.

40. Barragan P, Bouvier JL, Roquebert PO, et al. Resistance to thienopyridines: clinical detection of coronary stent thrombosis by monitoring of vasodilator-stimulated phosphoprotein phosphorylation. Catheter Cardiovasc Interv. 2003;59(3):295-302.

41. Bonello L, Camoin-Jau L, Arques S, et al. Adjusted clopidogrel loading doses according to vasodilator-stimulated phosphoprotein phosphorylation index decrease rate of major adverse cardiovascular events in patients with clopidogrel resistance: a multicenter randomized prospective study. J Am Coll Cardiol. 2008;51(14):1404-1411.

42. Sabatine MS, Hamdalla HN, Mehta SR, et al. Efficacy and safety of clopidogrel pretreatment before percutaneous coronary intervention with and without glycoprotein IIb/IIIa inhibitor use. Am Heart J. 2008;155(5):910-917.

43. Kastrati A, Mehilli J, Schuhlen H, et al. A clinical trial of abciximab in elective percutaneous coronary intervention after pretreatment with clopidogrel. N Engl J Med. 2004;350(3):232-238.

44. Kastrati A, Mehilli J, Neumann FJ, et al. Abciximab in patients with acute coronary syndromes undergoing percutaneous coronary intervention after clopidogrel pretreatment: the ISAR-REACT 2 randomized trial. JAMA. 2006;295(13):1531-1538.

45. Stone GW, McLaurin BT, Cox DA, et al. Bivalirudin for patients with acute coronary syndromes. N Engl J Med. 2006;355(21):2203-2216.

46. Lincoff AM, Steinhubl SR, Manoukian SV, Chew D, Pollack CV. Influence of timing of clopidogrel treatment on the efficacy and safety of bivalirudin in patients with non ST-segment elevation acute coronary syndromes undergoing percutaneous coronary intervention: an analysis of the ACUITY (Acute Catheterization and Urgent Intervention Triage Strategy) Trial. J Am Coll Cardiol Intv. 2008;1(6):639-648. 